Tema: Aciaria Oxigênio

\title{
DESENVOLVIMENTO DO CONTROLE DINÂMICO DOS CONVERTEDORES DA ACIARIA 1 UTILIZANDO A ANÁLISE DOS GASES*
}

\author{
Carlos André Ruy Carneiro ${ }^{1}$ \\ Hélio Alexandre Alves \\ João Domingos Guimarães Athayde Júnior ${ }^{3}$
}

\section{Resumo}

Em virtude da necessidade de produção de aços cada vez mais restritivos com relação às faixas de composição química, torna-se necessário o desenvolvimento contínuo de novas ferramentas para o controle do processo de fabricação do aço via aciaria à oxigênio. Neste cenário, propôs-se o desenvolvimento de um modelo de controle dinâmico para os Convertedores LD da Aciaria 1 da Usiminas - Usina de Ipatinga, utilizando o resultado da análise dos gases de exaustão, em conjunto com ferramentas matemáticas de ajuste e análise de dados. Neste trabalho é apresentado o modelo de previsão de carbono construído a partir das curvas das análises dos gases, das quais foram obtidos matematicamente os pontos de interesse: análises instantâneas, a interseção das curvas do $\mathrm{CO}$ e do $\mathrm{CO}_{2}$ e o ponto máximo da curva do $\mathrm{CO}_{2}$. $\mathrm{O}$ modelo foi desenvolvido utilizando redes neurais $\mathrm{e}$ validado utilizando dados históricos do processo.

Palavras-chave: Convertedor LD; Controle dinâmico; Análise dos gases; Redes neurais.

\section{DEVELOPMENT OF DYNAMIC CONTROL FOR CONVERTERS LD OF STEELMAKING SHOP 1 USING GAS ANALYSIS}

\section{Abstract}

Because of the need to produce increasingly restrictive steels with respect to chemical composition ranges, it is necessary to the continued development of new tools to control the meltshop process through oxygen steelmaking shop. In this scenario, it is proposed to develop a model of dynamic control for LD converters of Meltshop 1- Usiminas Ipatinga, using the result of analysis of the exhaust gases, together with mathematical tools for tuning and data analysis. This paper presents the model for predicting the carbon that was built starting from the curves of analyzes of gases, from which were mathematically obtained the interest points: the instant analysis, the intersection curves of $\mathrm{CO}$ and $\mathrm{CO}_{2}$ and the peak of the $\mathrm{CO}_{2}$ curve. The model was developed using neural networks and has been validated by historical data of the process.

Keywords: Blast oxygen furnace; Dynamic control; Analysis of gases; Neural network.

1 Engenheiro Metalurgista, Centro de Tecnologia Usiminas, Usiminas, Membro da ABM, Ipatinga, MG, Brasil.

2 Técnico Metalurgista, Centro de Tecnologia Usiminas, Usiminas, Ipatinga, MG, Brasil.

3 Engenheiro Metalurgista, M.Sc., Gerência Técnica Aciaria, Usiminas, Membro da ABM, Ipatinga, MG, Brasil.

\footnotetext{
* Contribuição técnica ao 450 Seminário de Aciaria - Internacional, 25 a 28 de maio de 2014, Porto Alegre, RS, Brasil.
} 


\section{INTRODUÇÃO}

Na etapa de sopro, a descarburação é a reação mais importante de uma aciaria a oxigênio, sendo também a reação mais amplamente estudada, tanto em escala laboratorial quanto industrial [1]. Uma correta predição da descarburação é a chave para o controle de fim de sopro, o que exige modelos bem ajustados. Os modelos devem, então, refletir a verdadeira natureza do fenômeno que se deseja modelar, contribuindo para o aumento do conhecimento sobre as reações processadas.

O acerto da composição química do aço e da temperatura no final do sopro de oxigênio são os fatores que definem o desempenho de uma planta de aciaria.

Uma das alternativas encontradas para atender as perspectivas de aumento de qualidade e produtividade é o desenvolvimento contínuo de novas ferramentas de controle de processo. Na Aciaria 1 da Usiminas - Usina de Ipatinga, existe somente o controle estático. Neste contexto, este estudo teve como objetivo principal desenvolver e implantar uma nova ferramenta de suporte para as decisões do soprador. Para isso, foram utilizados resultados da análise dos gases de exaustão do processo, visando à introdução de uma ferramenta de controle dinâmico.

\subsection{Aspectos Teóricos}

O controle dinâmico consiste na execução de cálculos de carga e condições de sopro antes do seu início, de acordo com os modelos de controle estático, e na coleta de informações durante o sopro, que permitem, por meio de modelos matemáticos, acompanhar a evolução do teor de carbono e a temperatura do banho. As ações corretivas são então estabelecidas, propiciando a obtenção de acerto simultâneo de carbono e temperatura.

Muitos estudos [1-3] vêm sendo realizados por empresas e institutos de pesquisa visando o desenvolvimento de sensores para aplicação do controle dinâmico, baseando na determinação da velocidade de descarburação por medição de vazão, temperatura e análise de gases, ou pelo controle da formação de escória através de detectores audiométricos. O método de controle dinâmico utilizando a sub-lança, desenvolvido no Japão, é o mais comum atualmente.

O controle dinâmico, baseado no uso da sub-lança, utiliza o resultado da medição de temperatura do banho e da estimativa do teor de carbono. Aproximadamente dois minutos antes do fim de sopro, a velocidade de descarburação deixa de ser máxima e constante tornando-se decrescente, em função da diminuição do teor de carbono no banho. O teor de carbono no início da redução da taxa de descarburação, carbon shift point (CSP), é um dos parâmetros utilizados nos modelos de controle dinâmico. Nesta fase do sopro, o oxigênio passa a reagir mais efetivamente com o ferro, tornando crescente a taxa de elevação da temperatura do banho. Outro parâmetro utilizado no controle dinâmico é o "HS", definido como um ajuste na curva calculada da taxa de elevação de temperatura $(\Delta \mathrm{T})$, visando atingir as taxas reais para as condições do processo naquele momento.

Um modelo de controle dinâmico com base nas análises contínuas dos gases de combustão do Convertedor oferece uma boa alternativa ao método que utiliza a sublança. O modelo de análise de gás pode melhorar o acerto no final de sopro e realizar o controle dinâmico ao longo do refino do aço, reduzindo, assim, projeções e formação de escória seca. Este método apresenta algumas vantagens, tais como resposta rápida, alta precisão e adaptabilidade no ambiente agressivo da aciaria.

\footnotetext{
* Contribuição técnica ao 450 Seminário de Aciaria - Internacional, 25 a 28 de maio de 2014, Porto Alegre, RS, Brasil.
} 
Diversos autores desenvolveram modelos com as informações geradas pelos gases de exaustão. Hahlin [3] utilizou a análise dos gases e balanço de massa para estimar o teor de carbono. Como este modelo é dependente do input de carbono, seja do gusa líquido ou outros insumos, este autor comenta que análises químicas confiáveis são particularmente difíceis de se obter e consequentemente, a previsibilidade do modelo pode ser comprometida.

Zhi-Gang [4] também desenvolveu um modelo de previsão de carbono final de sopro por meio do balanço de massa. O carbono residual no aço é calculado pela diferença entre o carbono inicial do processo (carga metálica e demais insumos) e o carbono total gerado nos gases, avaliado pela análise de $\mathrm{CO}$ e $\mathrm{CO}_{2}$. A descarburação é expressa pela Equação 1, baseada em medições constantes de $\mathrm{CO}$ e $\mathrm{CO}_{2}$ e na vazão de gás no sistema de exaustão.

$$
\frac{\mathrm{dc}}{\mathrm{dt}}=\frac{12}{22,4} \mathrm{Q}_{\mathrm{LDG}}[\varphi(\mathrm{CO}) \varphi(\mathrm{CO} 2)]
$$

Em que: dc/dt é a taxa de descarburação em $\mathrm{kg} / \mathrm{s} ; \mathbf{Q}_{\mathbf{L D G}}$ é a vazão no sistema OG em Nm $3 / s ; \varphi(\mathrm{CO})$ e $\varphi\left(\mathrm{CO}_{2}\right)$ são o volume percentual de $\mathrm{CO}$ e $\mathrm{CO}_{2}$ nos gases de exaustão.

Gomez [5] também cita que as vantagens do controle dinâmico por análise de gás são o aumento do nível de acerto do carbono, melhor controle do nível de oxidação do aço e da escória, permitindo reduzir o consumo de desoxidantes e ferros-liga, redução do tempo de sopro ao permitir o vazamento direto (sem análise química de laboratório), aumento de produtividade e redução de custos.

\subsection{Sistema de Análise de Gás da Usina de Ipatinga}

O sistema de exaustão de gases do Convertedor LD (OG) da Usiminas (Usina de Ipatinga) é mostrado esquematicamente na Figura 1. De acordo com Gomez [5], os gases gerados pelas reações do Convertedor saem a uma temperatura da ordem de $1600^{\circ} \mathrm{C}$, sendo, posteriormente, tratados e coletados.

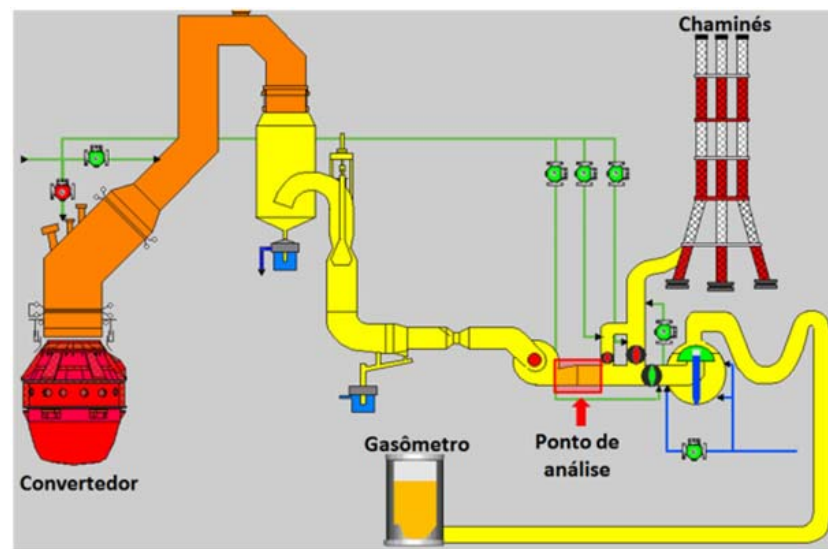

Figura 1. Desenho esquemático do sistema de exaustão de gases (Sistema OG) e destaque para o ponto de coleta e análise dos gases.

Atualmente na Usina de Ipatinga, a finalidade do sistema de análise do LDG é garantir que o gás gerado durante o sopro não contenha comburente e evitar que seja armazenado no gasômetro um gás com pouco poder calorífico. Para tal, o sistema atual realiza análises de $\mathrm{CO}, \mathrm{CO}_{2}, \mathrm{O}_{2}$ e $\mathrm{H}_{2}$, além da medição da vazão. Seguem, na Tabela 1, os limites e tipos de análises realizados.

* Contribuição técnica ao 450 Seminário de Aciaria - Internacional, 25 a 28 de maio de 2014, Porto Alegre, RS, Brasil. 
Tabela 1. Limites e tipos de análises para cada elemento/composto

\begin{tabular}{|c|c|c|}
\hline Compostos/Elementos & Limites (\%) & Método \\
\hline $\mathrm{CO} \mathrm{e} \mathrm{CO} 2$ & $0-100$ & Infravermelho \\
\hline $\mathrm{H}_{2}$ & $0-10$ & TC (Princípio da termo-condutividade) \\
\hline $\mathrm{O}_{2}$ & $0-25$ & Paramagnético \\
\hline
\end{tabular}

\subsection{Curvas das Análises dos Gases}

As reações de descarburação ocorrem durante a evolução do sopro e podem ser observadas por meio das medições contínuas de composição do gás de exaustão. Segue, na Figura 2, uma curva típica da taxa de descarburação obtida para uma corrida na Aciaria 1. Nota-se, claramente, que os pontos analisados representam as três etapas de descarburação.

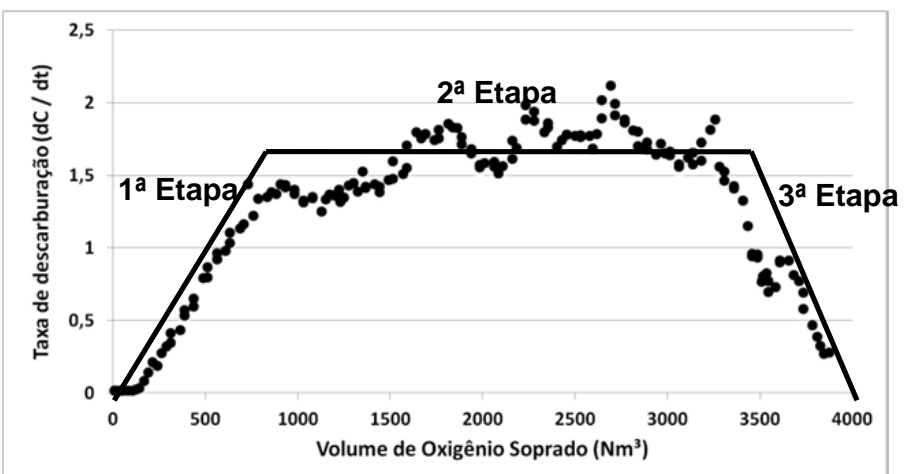

Figura 2. Curva característica da descarburação nos LDs da Aciaria 1.

Assim como mostrado na Figura 2, segue, na Figura 3, o perfil característico do comportamento da composição química dos gases durante o sopro.

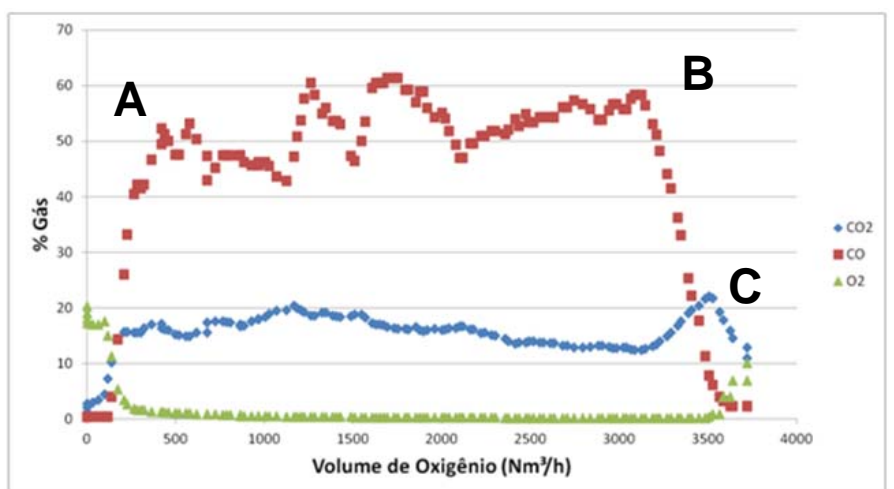

Figura 3. Perfil característico da composição do gás nos LDs da Aciaria 1.

Conforme Gomez [5], a curva para o CO mostra um período em que a concentração é mantida aproximadamente constante. Esta etapa do processo (cujo ponto de partida é indicado com a letra A e termina com o ponto indicado por B - Figura 3) representa o período de descarburação máxima do banho. Nesta fase, praticamente todo o oxigênio soprado pela lança é utilizado na reação de descarburação. No final, há um aumento na concentração de $\mathrm{CO}_{2}$, que atinge um máximo (ponto $\mathrm{C}$ - Figura 3) e depois volta a reduzir. Durante este período, a taxa de descarburação diminui e, por conseguinte, uma parte do oxigênio reage com $\mathrm{CO}$, para formar $\mathrm{CO}_{2}$. Ao mesmo tempo, o ar ambiente introduzido no Convertedor favorece a reação de póscombustão de $\mathrm{CO}$, sendo convertido em $\mathrm{CO}_{2}$. Estes fatos explicam o aumento

* Contribuição técnica ao 45 Seminário de Aciaria - Internacional, 25 a 28 de maio de 2014, Porto Alegre, RS, Brasil. 
observado na curva de $\mathrm{CO}_{2}$, acompanhado por redução da concentração de $\mathrm{CO}$. Quando o fluxo de gás é suficientemente reduzido, todo o $\mathrm{CO}$ é convertido em $\mathrm{CO}_{2}$, obtendo-se o máximo da curva de $\mathrm{CO}_{2}$. A partir deste ponto, segue diminuindo o volume de gases gerados durante a descarburação, aumentando os teores e a representatividade do $\mathrm{O}_{2}$ nos valores totais.

\subsection{Redes Neurais Artificiais (RNAs)}

RNAs são sistemas paralelos, distribuídos, compostos por unidades de processamento simples (nodos) que calculam determinadas funções matemáticas (normalmente não lineares). Tais unidades são dispostas em uma ou mais camadas e interligadas por um grande número de conexões, geralmente unidirecionais. $\mathrm{Na}$ maioria dos modelos, estas conexões estão associadas a pesos, os quais armazenam o conhecimento representado no modelo e servem para ponderar a entrada recebida por cada neurônio da rede. O funcionamento destas redes é inspirado em uma estrutura física concebida pela natureza: o cérebro humano [6]. A solução de problemas através de RNAs é bastante atrativa, pois, devido à forma como estes são representados internamente pela rede e o paralelismo natural inerente à sua arquitetura criam a possibilidade de um desempenho superior ao dos modelos convencionais. O procedimento usual na solução de problemas passa inicialmente por uma fase de aprendizagem, em que um conjunto de exemplos é apresentado para a rede, a qual extrai automaticamente as características necessárias para representar a informação fornecida. Estas características são utilizadas posteriormente para gerar respostas para o problema [6].

A capacidade de aprender por meio de exemplos e de generalizar a informação aprendida é, sem dúvida, o atrativo principal da solução de problemas através de RNAs. A generalização, que está associada à capacidade da rede aprender através de um conjunto reduzido de exemplos e, posteriormente, dar respostas coerentes para dados não conhecidos, é uma demonstração de que sua capacidade vai muito além do que simplesmente mapear relações de entrada e saída. As RNAs são capazes de extrair informações não apresentadas de forma explícita através dos exemplos. Não obstante, também são capazes de atuar como mapeadores universais de funções multivariáveis, com custo computacional que cresce linearmente com o número de variáveis. Outra característica importante é a capacidade de auto-organização e de processamento temporal, que, aliada àquelas citadas anteriormente, faz das RNAs uma ferramenta computacional bastante útil.

\section{MATERIAIS E MÉTODOS}

O escopo do estudo abrangeu os Convertedores da Aciaria 1. Estes reatores contam somente com o controle estático do processo, resultando em um processo com maior criticidade no que diz respeito ao acerto simultâneo.

Para o desenvolvimento do modelo de controle dinâmico, as concentrações percentuais de $\mathrm{CO}, \mathrm{CO}_{2}$ e $\mathrm{O}_{2}$ foram coletadas e tratadas estatisticamente. Tais informações coletadas foram referentes aos dados instantâneos e os dois pontos previamente definidos das curvas das análises de gás. Tais pontos foram:

- $\quad$ a interseção das curvas de $\mathrm{CO}$ e $\mathrm{CO}_{2}$; e

- $\quad$ o ponto máximo da curva de $\mathrm{CO}_{2}$.

Após a construção do banco de dados, foram retirados os dados que estatisticamente se apresentaram fora dos limites aceitáveis (outliers). Esta prática

\footnotetext{
* Contribuição técnica ao 45 Seminário de Aciaria - Internacional, 25 a 28 de maio de 2014 Porto Alegre, RS, Brasil.
} 
auxilia na remoção de parâmetros incorretos que podem influenciar positivamente (um outlier pode aumentar o coeficiente de correlação, mas mesmo assim a influência dele no modelo é negativa, pois não estará representando as condições reais) ou negativamente nos ajustes estatísticos propostos.

O método adotado para esta remoção de outliers foi por meio da construção e análise dos quartis (distribuição) dos valores de cada variável. Uma restrição para esta metodologia é que a variável em questão deve apresentar distribuição normal. Logo, todas as variáveis também serão testadas quanto a este ponto.

Primeiramente, foram determinados os quartis e a amplitude interquartil (IQR), como demonstrado na Figura 4, para posterior filtro dos pontos considerados outliers.
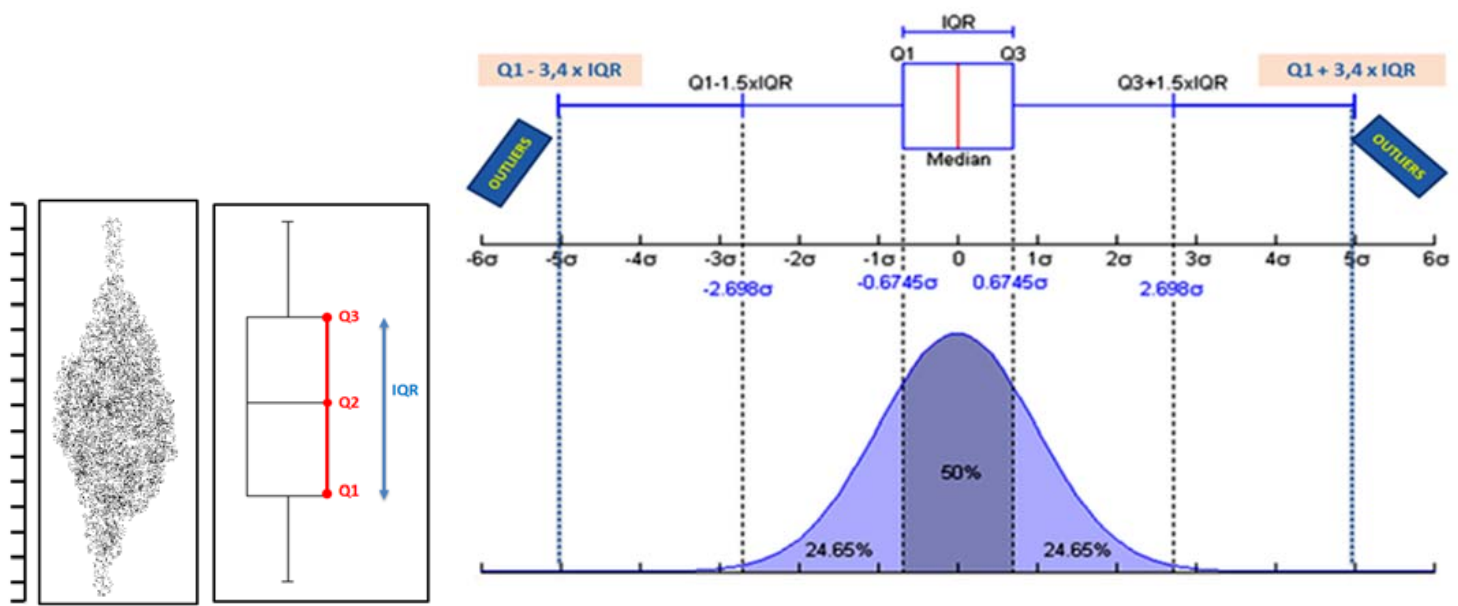

Figura 4. Determinação dos quartis por meio da distribuição dos dados.

Com base na análise realizada, na fundamentação teórica do problema e revisão bibliográfica, foram identificadas as variáveis a serem utilizadas no desenvolvimento da RNA. O software utilizado para o desenvolvimento da rede foi o Matlab.

A definição de uma equação que representa os fenômenos do processo foi a primeira etapa para a aplicação industrial. Após este passo, o modelo foi validado na área operacional de forma off-line utilizando dados históricos do processo.

\section{RESULTADOS E DISCUSSÃO}

Para desenvolvimento da RNA, foram realizadas diversas simulações com objetivo de identificar as variáveis que apresentaram as melhores correlações. Para isso, foram coletados dados de 318 corridas do Convertedor 2 da Aciaria 1.

A RNA desenvolvida foi do tipo MLP (Multiplayer perceptron), cuja característica é apresentar pelo menos uma camada intermediária ou oculta. Posteriormente, foi implementado o algoritmo de treinamento back-propagation com parada antecipada (regularização), utilizando a função tansig e a normalização com premnmx.

No treinamento de um perceptron de múltiplas camadas com o algoritmo de backpropagation é relativamente difícil identificar o melhor momento de encerrar o treinamento, comprometendo a generalização do modelo. Nestes casos, a rede acaba sendo ajustada apenas para os dados de treinamento (supertreinamento). Usando a técnica da validação cruzada, o subconjunto de estimação é usado para treinar a rede, sendo o treinamento periodicamente interrompido para avaliação da rede com o subconjunto de validação. Este processo de treinamento seguido de

* Contribuição técnica ao 450 Seminário de Aciaria - Internacional, 25 a 28 de maio de 2014, Porto Alegre, RS, Brasil. 
validação é conhecido como "Método de Parada Antecipada". No presente trabalho, foi determinada a relação $75 \% / 25 \%$ entre treinamento e validação, respectivamente. O algoritmo de otimização escolhido para o modelamento foi o LevenbergMarquardt. Ressalta-se que o tipo de treinamento Back Propagation, onde a função erro a ser minimizada é não linear e no formato de mínimos quadrados, favorece o desempenho e a aplicação deste algoritmo.

Por fim, para evitar o underfitting e o overfitting, foi inserido na programação um looping para determinação do número ideal de neurônios na camada oculta. Desta forma, visou-se encontrar a melhor configuração da rede para cada caso em específico.

Foram identificados oito variáveis que apresentaram boa correlação para a previsão do carbono final de sopro. Tais variáveis são descritas na Tabela 2.

Tabela 2. Variáveis da rede neural.

\begin{tabular}{|l|l|l|}
\hline Variável & Unidade \\
\hline Var1 & Gusa Líquido & $\mathrm{Kg}$ \\
\hline Var2 & Nível do banho & $\mathrm{mm}$ \\
\hline Var3 & Volume de Oxigênio entre "Max $\mathrm{CO}_{2}$ " e final do sopro & $\mathrm{Nm}^{3}$ \\
\hline Var4 & $\mathrm{CO}$ & $\%$ \\
\hline Var5 & $\mathrm{CO}_{2}$ & $\%$ \\
\hline Var6 & $\mathrm{O}_{2}$ & $\%$ \\
\hline Var7 & dC/dt & $\% / \mathrm{s}$ \\
\hline Var8 & Volume de Oxigênio entre os pontos "A" e "B" & $\mathrm{Nm}^{3}$ \\
\hline
\end{tabular}

Um ponto identificado no desenvolvimento do modelo, e que deve ser levado em consideração, é a condição de funcionamento do sopro combinado (KGC). Tal parâmetro, por ter influência na taxa de descarburação, foi determinante para a definição dos pesos da rede. Nesse sentido, foi desenvolvido um modelo considerando o KGC em operação padrão e outro para quando o KGC não estiver operando na sua condição padrão.

Na Figura 5 são apresentadas as curvas de composição química dos gases gerados no Convertedor ao longo do sopro indicando cada variável utilizada como entrada da rede desenvolvida.

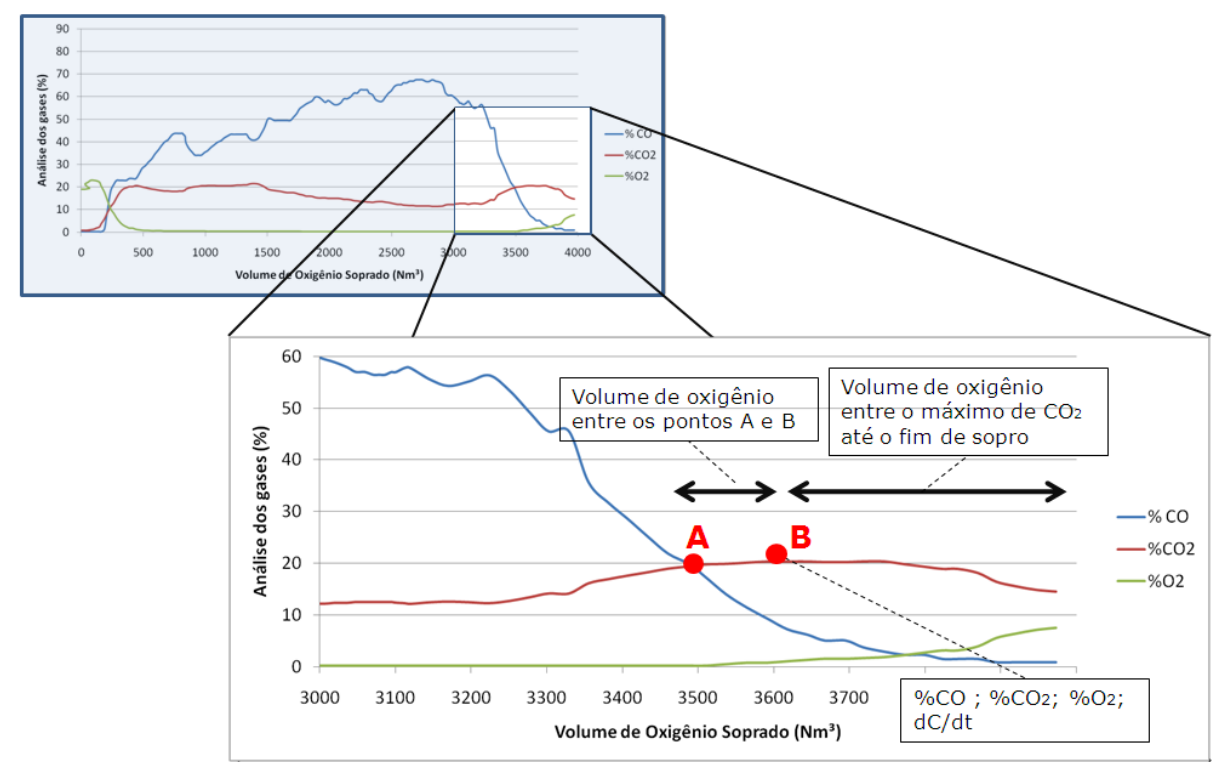

Figura 5. Visão gráfica das variáveis consideradas no modelo.

\footnotetext{
* Contribuição técnica ao 45 Seminário de Aciaria - Internacional, 25 a 28 de maio de 2014 Porto Alegre, RS, Brasil.
} 
O modelo com os melhores resultados nas validações off-line apresentou a seguinte concepção:

- A partir de $77 \%$ do início do sopro iniciam-se os cálculos gerando os teores instantâneos de carbono do banho;

- Assim como os valores instantâneos da composição dos gases, os volumes referentes aos pontos A e B (apresentados na Figura 5) são armazenados e alimentados na rede neural;

- A partir do ponto $B$, a única variável em aberto é o volume de oxigênio, todas as outras ficam congeladas. Com isso, abre a possibilidade de obter o carbono instantâneo e uma previsão de carbono final para $\mathrm{Nm}^{3}$ de oxigênio a ser soprado. Tal fato justifica o nome da ferramenta como controle dinâmico, pois concilia valores instantâneos com previsões de carbono no fim de sopro.

Os resultados das redes com e sem o KGC seguem nos itens abaixo.

\subsection{Rede com o KGC operando}

O treinamento da rede foi realizado com 165 corridas. O modelo que apresentou o melhor resultado foi com 10 neurônios na camada oculta. Segue na Figura 6 a relação entre o carbono previsto e o real.

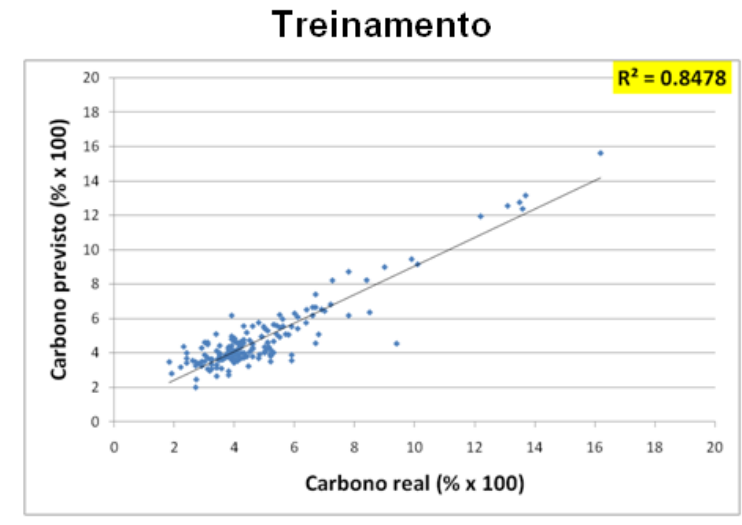

(a)

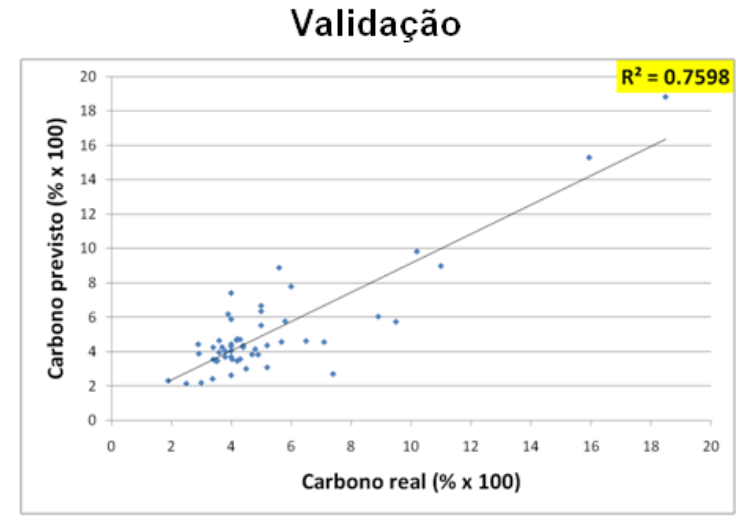

(b)

Figura 6. Resultado do treinamento e validação da rede neural.

A análise estatística (ANOVA) realizada para os dados apresentados na Figura 6(a) apresentam um $\mathrm{P}$-valor inferior a 0,01 , indicando que existe uma relação estatisticamente significativa entre o carbono previsto e o real, com nível de confiança de $99 \%$. Além disso, o índice de correlação quadrático $\left(R^{2}\right)$ afirma que o modelo explica $85 \%$ da variabilidade dos resultados reais. O coeficiente de correlação apresentou o valor de 0,92 , indicando uma forte relação entre as variáveis.

Em relação aos dados de validação, na figura 6(b), uma vez que o P-valor é inferior a 0,01 , existe uma relação estatisticamente significativa entre o carbono previsto e o real, no nível de confiança de $99 \%$. O índice de correlação quadrático $\left(R^{2}\right)$ afirma que o modelo explica $76 \%$ da variabilidade dos resultados reais. O coeficiente de correlação apresentou o valor de 0,87 , indicando uma forte relação entre as variáveis.

\footnotetext{
* Contribuição técnica ao 45 Seminário de Aciaria - Internacional, 25 a 28 de maio de 2014, Porto Alegre, RS, Brasil.
} 


\subsection{Rede sem o KGC Operando}

O treinamento da rede foi realizado com 153 corridas. O modelo que apresentou o melhor resultado foi com 5 neurônios na camada oculta. A validação da rede foi realizada com 50 corridas. É apresentada na Figura 7 a relação entre o carbono previsto e o real.

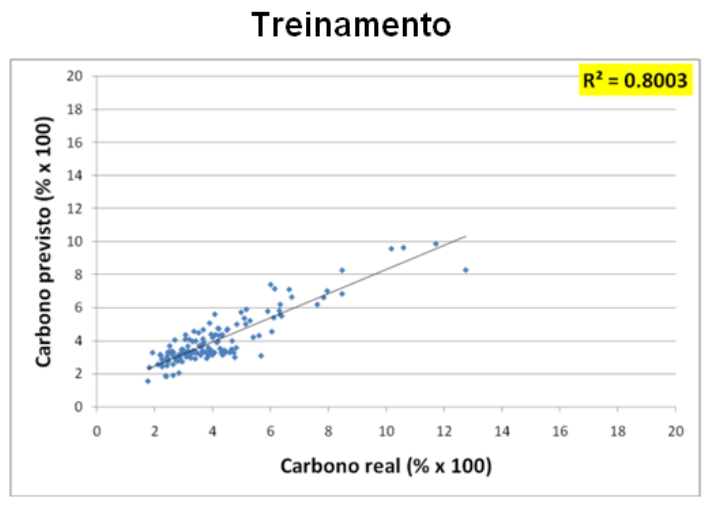

(a)

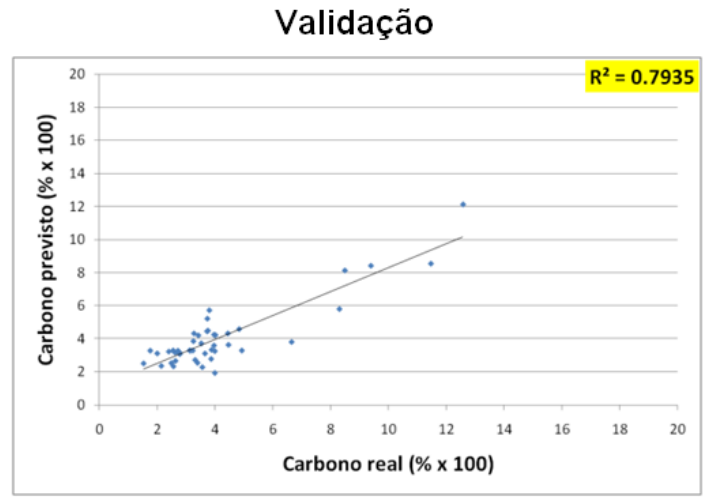

(b)

Figura 7. Resultado do treinamento e validação da rede neural.

Da mesma forma que realizado anteriormente, a análise estatística (ANOVA) realizada para os dados apresentados na Figura 7a apresentam um P-valor inferior a 0,01 , indicando que existe uma relação estatisticamente significativa entre o carbono previsto e o real, com nível de confiança de $99 \%$. O índice de correlação quadrático $\left(R^{2}\right)$ afirma que o modelo explica $80 \%$ da variabilidade dos resultados reais. $O$ coeficiente de correlação apresentou o valor de 0,89 , indicando uma forte relação entre as variáveis.

Em relação aos dados de validação, na Figura $7 \mathrm{~b}$, uma vez que o P-valor na tabela ANOVA é inferior a 0,01, existe uma relação estatisticamente significativa entre o carbono previsto e o real, no nível de confiança de $99 \%$. O índice de correlação quadrático $\mathrm{R}^{2}$ afirma que o modelo explica $79 \%$ da variabilidade dos resultados reais. O coeficiente de correlação apresentou o valor de 0,89 , indicando uma forte relação entre as variáveis.

Em linhas gerais, na Figura 8 são apresentados os erros absolutos dos modelos (diferença entre os valores reais e estimados).

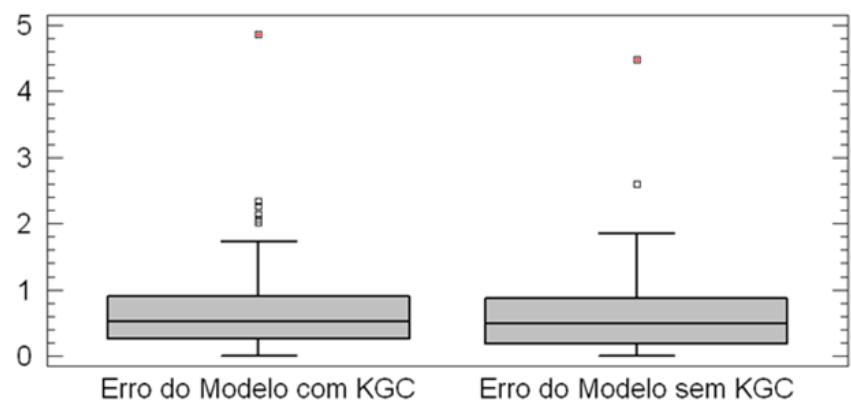

\begin{tabular}{|c|c|c|}
\hline \multicolumn{3}{|c|}{ Erro Absoluto (\% x 100) } \\
\hline Modelo & Média & $\begin{array}{c}\text { Desvio } \\
\text { padrão }\end{array}$ \\
\hline $\begin{array}{c}\text { Com } \\
\text { KGC }\end{array}$ & 0,66 & 0,61 \\
\hline $\begin{array}{c}\text { Sem } \\
\text { KGC }\end{array}$ & 0,60 & 0,56 \\
\hline
\end{tabular}

Figura 8. Resultado do treinamento e validação da rede neural.

O erro absoluto de ambos os modelos foi de aproximadamente 0,6 pontos de carbono $(\% \times 100)$. Valores considerados baixos e que proporcionam ao processo um controle confiável.

* Contribuição técnica ao 45 Seminário de Aciaria - Internacional, 25 a 28 de maio de 2014, Porto Alegre, RS, Brasil. 
De forma simplificada, seguem alguns passos realizados pelo modelo para previsão do carbono final de sopro.

\subsubsection{Passo 1: entrada do volume de $\mathrm{O}_{2}$ previsto}

O volume de oxigênio previsto pelo cálculo estático do modelo dos Convertedores define o início da faixa em que o modelo dinâmico poderá estimar com confiabilidade, o carbono instantâneo de sopro. Foi definido que esta previsão do carbono instantâneo inicia a partir de $77 \%$ do volume de oxigênio total previsto.

\subsubsection{Passo 2: cálculo da taxa de descarburação}

A taxa de descarburação (dC/dt) é calculada conforme a Equação 2.

$$
\frac{\mathrm{dC}}{\mathrm{dt}}=\left(0,0001488 \mathrm{Q}_{\mathrm{LDG}}\right)(0,0042857 \% \mathrm{CO}+0,0027273 \% \mathrm{CO})
$$

Sendo:

$Q_{\text {LDG }}=$ Vazão do LDG $\left(\mathrm{Nm}^{3} / \mathrm{h}\right)$

\subsubsection{Passo 3: identificação dos pontos "A" e "B"}

São identificados os intervalos característicos que serão alimentados na rede neural, denominados pontos "A" e "B".

O ponto " $A$ " refere-se ao volume de oxigênio soprado entre a intercessão das curvas de $\mathrm{CO}$ e $\mathrm{CO}_{2}$ e ao valor máximo de $\mathrm{CO}_{2}$, o ponto "B" refere-se ao volume de oxigênio entre o valor máximo de $\mathrm{CO}_{2}$ e o final do sopro, conforme Figura 5.

\subsubsection{Passo 4: alimentação do modelo}

As variáveis listadas na tabela 2 são alimentadas no modelo das redes neurais.

Após o ponto $B$, todas as variáveis permanecem inalteradas, apresentando os mesmos valores. Somente a VAR3 (volume de oxigênio entre "Max $\mathrm{CO}_{2}$ " e final) fica aberta e alterando até o final do processo.

\subsubsection{Passo 5: rede neural}

Conforme mencionado anteriormente, são dois os modelos de redes neurais desenvolvidos. Se o KGC estiver em funcionamento normal é considerado o modelo 1 , caso não esteja funcionando é considerado o modelo 2.

A alimentação das variáveis no modelo devem seguir os seguintes cálculos:

a) Normalização dos dados de entrada:

$$
\text { Normalização }=\frac{2 \text { (Variavel-Faixa mínima })}{(\text { Faixa máxima-Faixa mínima })}-1
$$

b) Cálculo do somatório dos bias e pesos para cada neurônio, conforme equação 3:

$$
\text { Somatório }=(\mathrm{Xi} \mathrm{Wi})+\mathrm{Bi}
$$

c) Aplicação da função de ativação em cada neurônio:

$$
\text { Função Ativação = 2/[1+EXP(-2 Somatório) }] \text { - } 1
$$

d) Somatório de todos os valores encontrados no item c:

$$
\text { Somatório2 }=\Sigma F A
$$

e) Aplicação da função de ativação para o valor encontrado no item d:

$$
\mathrm{FA}_{2}=2 /[1+\mathrm{EXP}(-2 \text { Somatório2) }]-1
$$

f) Desnormalização do resultado encontrado:

$$
\mathrm{CFS}=0,5(\text { Var+1) } \text { (Faixa Máxima - Faixa Mínima) + Faixa Máxima }
$$

\footnotetext{
* Contribuição técnica ao $45^{\circ}$ Seminário de Aciaria - Internacional, 25 a 28 de maio de 2014, Porto Alegre, RS, Brasil.
} 


\section{CONCLUSÕES}

Nesse trabalho foram apresentadas as considerações relativas ao desenvolvimento de um modelo de previsão de carbono durante o sopro de oxigênio no Convertedor LD, assim como uma ferramenta para o controle dinâmico do processo em função dos valores finais almejados. Em virtude dos resultados alcançados, fica comprovada a viabilidade da utilização dos gases de exaustão para o controle do processo, sem a necessidade de maiores investimentos.

Após todas as análises, foi identificada a melhor estrutura lógica do modelo. Alguns pontos importantes definidos foram: somente após $77 \%$ do sopro, a rede neural é alimentada pela análise química dos gases, gerando como resultado o valor instantâneo do carbono; o volume dos gases no ponto de interseção das curvas $\mathrm{CO} / \mathrm{CO}_{2}$ e no ponto máximo da curva do $\mathrm{CO}_{2}$ são identificados e também alimentados na rede neural; e, após a ocorrência do ponto máximo na curva do $\mathrm{CO}_{2}$, somente as informações do volume de oxigênio soprado são levadas em consideração para o cálculo do carbono instantâneo e o carbono final de sopro previsto.

A configuração da rede neural proposta, assim como sua programação, se mostrou eficiente e atendeu o objetivo, considerando que os modelos gerados apresentaram boa confiabilidade e previsibilidade. Os índices de correlações das curvas foram considerados suficientes para a aplicação proposta.

Esperam-se potenciais ganhos em produtividade, após a completa implementação do modelo na área industrial, através do aumento do acerto do carbono visado, redução do índice de ressopro, redução da oxidação final do banho e consequentemente redução do consumo de desoxidantes, aumento da vida dos refratários, dentre outros.

\section{REFERÊNCIAS}

1 Etrusco GSP. Controle dinâmico do processo LD. In: $29^{\circ}$ Congresso Anual da ABM; 1984; Belo Horizonte, Brasil. São Paulo: ABM; 1984.

2 Viana JF, Castro LFA. Utilização de rede neural na modelagem de sopro do Convertedor LD. In: $34^{\circ}$ Seminário de Fusão, Refino e Solidificação dos Metais; 2003; Belo Horizonte, Brasil. São Paulo: ABM; 2003. p.27-36.

3 Hahlin P. A dynamic system for converter control in steelmaking. Redhill: Steel times International - supplement; jun.1993.

4 Zhi-Gang BY, Liu L, Ping H, Xiang TM. A dynamical off-gas model on a 150t BOF. Redhill: Steel times International; abr./mai.; 2003.

5 Gomez A, Data A, Tomassini G, Cicutti C, Bertezzolo U, Donayo R. Determinacion del contenido de carbono al fin de soplo de lós convertidores en función de los gases de salida. In: 16th IAS Steelmaking Conference; 2007; Rosario, Argentina.

6 Braga AP, Ludermir TB, Carvalho ACPLF. Redes neurais artificiais, teoria e aplicações. São Paulo: Editora LTC; 2000.

* Contribuição técnica ao 45 Seminário de Aciaria - Internacional, 25 a 28 de maio de 2014, Porto Alegre, RS, Brasil. 